\title{
Solving RFID mobile reader path problem with optimization algorithms
}

\author{
M. Zaki Zakaria ${ }^{1}$, Sofianita Mutalib ${ }^{2}$, Shuzlina Abdul Rahman ${ }^{3}$, Shamsul J Elias ${ }^{4}$, \\ A Zambri Shahuddin 5 \\ 1,2,3,5 Faculty of Computer and Mathematical Sciences, University Technology of MARA, Malaysia \\ ${ }^{4}$ Faculty of Computer and Mathematical Sciences, University Technologi MARA Kedah, Malaysia
}

\section{Article Info}

Article history:

Received Oct 5, 2018

Revised Dec 6, 2018

Accepted Dec 17, 2018

\section{Keywords:}

$\mathrm{ACO}$

GA

Path optimization

PSO

RFID

\begin{abstract}
Radio Frequency Identification (RFID) is a one of the fastest growing and most beneficial technologies being adopted by businesses today. One of the important issues is localization of items in a warehouse or business premise and to keep track of the said items, it requires devices which are costly to deploy. This is because many readers need to be placed in a search space. In detecting an object, a reader will only report the signal strength of the tag detected. Once the signal strength report is obtained, the system will compute the coordinates of the RFID tags based on each data grouping. In this paper, algorithms using genetic algorithm, particle swarm, ant colony optimization are proposed to achieve the shortest path for an RFID mobile reader, while covering full search area. In comparison, for path optimization, the mobile reader traverses from one node to the next, moving around encountered obstacles in its path. The tag reading process is iterative, in which the reader arrives at its start point at the end of each round. Based on the shortest path, an algorithm that computes the location of items in the search area is used. The simulation results show that the ACO method works more effectively and efficiently compare to others when solving shortest path problems.
\end{abstract}

Copyright $\odot 2019$ Institute of Advanced Engineering and Science. All rights reserved.

\section{Corresponding Author:}

M. Zaki Zakaria,

Faculty of Computer and Mathematical Sciences,

University Technology of MARA,

Shah Alam, Malaysia.

Email: fmzaki@yahoo.com

\section{INTRODUCTION}

With the growth of the Internet of Things (IOT), the Radio Frequency Identification (RFID) will be the most important technology to be adopted and it will be seen as the pre-requisite for the IOT. The RFID uses radio waves to identify people or objects, with applications in contactless payment, asset management, trusted identification (ID) and document tracking [1]. Based on the market observation by Frost and Sullivan, the Malaysian RFID market in 2009 was estimated to be approximately RM36 million and has been expected to grow to about RM120 million in 2020. The RFID deployment will grow rapidly due to the extensive implementation of the IOT, thus it is essential for the RFID technology to embed the capability to trace any object easily once it is tagged. In general, RFID is able to tag, trace and check an object, and RFID tags are attached to objects. The tags captured important information about an object, such as its unique ID number, manufacture date and product composition.

RFID technology uses radio waves to enable the communication between readers and tags. The RFID tag is a device that stores an object's data such as its ID, and transmits it to the reader in a contactless manner using radio waves. Objects are identified when a tag transmits this information back to the readers within the interrogation range. The reader is a device that can read data from and write data to 
compatible RFID tags. RFID readers can be fixed (static), or mobile (within a predefined physical area). Objects are identified when tags transmit this information back to the readers within the interrogation range.

Based on the analysis and the forecast conducted by IDTechEx (a research company based in the United Kingdom which has tracked the RFID market since 1999), RFID will continue to be adopted in retail industry. In 2016 alone, the demand forecasted for apparel tagging was 4.6 billion RFID labels while the RFID in the form of tickets used for transit would demand 800 million tags ready for consumption. The tagging of animals (such as pigs, sheep and pets) is substantial as it continues to be a legal requirement in many more territories, with 420 million tags being used for this sector in 2016. In total, IDTechEx expects those 8.9 billion tags to be sold in 2015 and 10.4 billion in 2016. Based on the forecast, RFID deployment in Malaysia will be the biggest potential contributor to the revitalizing of the electronics and electrical sector as aimed under the Economic Transformation Plan. To achieve this, it is important to identify the key factors in ensuring the affordability for the mass implementation.

In this paper, we propose Genetic Algorithm (GA), Particle Swarm Optimization (PSO) and Ant Colony Optimization (ACO) techniques that incorporate the nearest neighbor for the movement of RFID mobile readers. The optimum path (i.e., shortest path) information obtained is used to compute the location of objects within the given area using the triangulation technique.

It has always been our intention to discover the best technique in locating the RFID readers (nodes) within a given area to guarantee $100 \%$ coverage by employing a minimum number of readers. We develop a prototype of the software simulation to determine how many RFID fixed readers are required to cover a given area based on hexagonal packing in our first phase. One RFID reader is able to cover every hexagon area and the coverage of a wireless sensor network may be approximated by a disk of a prescribed radius (sensing range). Coverage can be computed by taking the union of individual coverage areas of all sensors (readers) in the network. This can be performed by assuming that each circle is a hexagon which attaches with another. We assume all the readers have the same sensing and transmission range.

In the second phase, the location of the RFID reader that we calculated previously is used as a reference point the mobile reader visits in a certain period of time. We proposed to use the mobile RFID reader to reduce hardware (reader) costs, and eliminate readers' interference. The mobile reader is moved from one point (node) to another point based on the shortest path computed using PSO and ACO for the given dimension area. The tag reading process by the mobile RFID reader is repeated when the reader arrives to its start node in the loop. This process repeated in an iterative fashion.

In the third phase, we start the algorithm with an initialization process to determine the number of tags to be used in the given area. The process continues by placing tags at random in the same area. The process continues which tags detecting by mobile RFID reader 3 times at different location or point in the given dimension area. The process continues by detecting a reader and it is done until all three readers are detected the tags. Then we continue with the calculation to determine the position of tags by using the equations discussed in Section 3. The equation is based on the data of the received signal strength indication (RSSI) that is obtained during the detection process. This paper presents an algorithm for the RFID mobile reader for path optimization using GA, PSO and ACO techniques incorporating the nearest neighbor which is the algorithm is able work with any design area, whether it is asymmetrical or symmetrical. In addition, the algorithm has the ability to define the position of the obstacles based on working environment.

\section{RELATED WORKS}

RFID technology is used in many applications for example in tracking or identifying objects [2] measure data of proximity, Received Signal Strength (RSS) and read rate of passive RFID tags by simulation modelling. The comparison of localization accuracy using different algorithms shown that results on average is $8 \%$. A localization technique has been proposed using combination of wireless sensor network (WSN) and radio frequency identification technology to solve the localization in WSN [3]. UHF RFID transponders and Direction of Arrival (DoA) estimation technique has been used to detect tags localization by filtering out the carrier frequency and the signal is used for estimating the direction of arrival [4]. A localization methods using Phase Different of Arrival (PDoA) has been proposed [5]. Moreover, various gaps in the approaches based on PDoA are identified. RFID-based centralized cooperative localization has been suggested in indoor environment [6]. The method is to improve the positioning accuracy of four users moving in a building, by using the received signal strength (RSS) measurements from a number of known location RFID tags (anchors) and combining it with RSS measurements of mobile tags carried by themselves.

The principle of the Landmarc approach was used to introduce the concept of proximity map [7], which is the whole sensing area is divided into regions, where each region corresponds to a reference tag. A probabilistic localization technique has been suggested to detect the object using three steps [8]. First, the propagation variables are calibrated using on-site reference tags, and then the distance between the 
object (targeted tag) and the readers is estimated with a probabilistic RSS model. Finally, the location of the tag is determined by applying a Bayesian inference technique.

RFID technology has also been used to guide blind people to find the shortest route from their current location to a destination. This technology also helps them when they get lost by automatically detecting the route, and recalculating a new route to the same destination [9]. The system embeds RFID tags into a footpath that can be read by an RFID reader with a cane antenna. The system is also used for navigation in rescue operations involving hazardous environments, where it is difficult to find an emergency exit. The indoor guidance system [10] uses RFID technology to find a guidance path for important places, such as museums, hospitals, airport terminals and exhibition halls.

The most challenging task is to achieve the shortest path for a network by minimizing the cost (distance) inside a given area. The shortest path has been used extensively for other purposes, such as vehicle routing in the transportation system [11], path planning in robotics [12] and traffic routing in communication networks [13]. An application for complete coverage within a predefined period of time using mobile RFID readers in a symmetrical area has been introduced [14]. The system proposed by [14] only catered for symmetrical area, and within the area covered by RFID mobile readers, she assumes the areas only locate the proper stacked shelves. The given area is divided into many sectors, and each sector is catered by one mobile reader. The path shown in [14] is similar for every sector. [15] presents the investigation of PSO to solve the shortest path routing problem using a modified priority based indirect encoding, incorporating a heuristic operator for reducing the possibility of loop formation in the path construction process.

\section{PATH OPTIMIZATION}

The shortest path problem is finding a path between nodes in a search space or area such that the sum of the weights or distance of edges is minimized. The problem of finding the minimum distance for this case can be modeled by assuming that each node in a search space (such that the sum of distance of its constituent edges) is minimized. There are many researchers that have been done in the shortest path problem but not in path optimization for mobile RFID reader [16]. A simulation is created to test for the path optimization such as ACO, PSO and GA. Simulations are conducted to compare results for all techniques.

\subsection{Genetic Algorithm}

In this work, a basic GA is employed to solve the benchmark problem for path optimization of mobile RFID reader. The GA represents the design variable with nodes number that referred to as chromosomes. The GA works for a combination of discrete parameters and also forces the design variables to only take values with no duplicate values i.e. no solution is ever produce the duplicate node in new chromosomes. One population from solutions are used to form a new population with the hope that the new population may be better than the old one. The population which is selected to form new solutions (chromosome @ offspring) must meet the fitness criteria in which, the fitter they are, the more chances to reproduce. This process repeats continuously until the condition is satisfied such as achieving the total number of iteration.

The process starts from a population of randomly generated individual and each iteration is called a generation. In every generation, the fitness of each chromosome is calculated based on objective function in path optimization (minimum path) for a mobile RFID reader. The new generation of chromosome simply crossed to produce offspring tour which results in illegally tour i.e. tours which visit some nodes more than one time. To deal with this problem, the offspring tours are corrected so that the duplicate nodes are replaced by unvisited nodes at random. The process terminates when a maximum number of iteration has been produced or the result of fitness function is satisfactory.

In the first case, the algorithm randomly initializes population of chromosome. For example, eight (8) locations (nodes) of a searched space are identified in which the mobile RFID reader will be visited not more than once and the number of population are five. Next step is to apply the nearest neighbour constraint in which to ensure that the next node to go is in the surrounding list of current nodes. With this application, user can choose a level of the nearest neighbour. For level 1, surrounding nodes is within radius of current node coverage (mobile reader coverage) but for level 2 surrounding node is double of reader coverage (node). The reason to implement the level of the nearest neighbour is to control the new chromosome with the valid path results.

Based on the solutions shows on Table 4, there are four (4) valid populations which are all nodes visited only once and there is one solution that is invalid because the surrounding nodes (neighbour nodes) have been visited more than once. Chromosome 2 and 3 have the best value for fitness function (minimum distance) and these chromosomes are then being treated as parent for the next iteration. The next step is to crossover by creating new population which uses one-point crossover technique. In this phase, the offspring 
will be checked so that no duplicate value in the operators. Duplicate value will be replaced by unvisited nodes at random. Next phase is mutation process in which the operator is randomly picked. The main reason for mutation is to preserve the genetic diversity (exploitation) of the population. The process continues until the termination criteria are satisfied and when specific number of iteration has been exceeded. Although sometimes the performance of an algorithm is observed to be satisfactory, still it encounters some faults, such as the local search ability and the genetic algorithm does not assure to find a global optimum.

\subsection{Particle Swam Optimization}

Particle swarm optimization (PSO) is a population-based stochastic optimization technique that originates from nature and evolutionary computations, developed by [17]. The PSO is a method that is able to identify an optimal solution within a population (i.e., a swarm). The PSO algorithm flow consists of a population of individuals referred to as "particles". Every particle is a potential solution to an n-dimensional problem. The group can achieve the solution effectively by using common information shared by the group, and the information is owned by the particle itself. The particles change their state by "flying" around in an n-dimensional search space based on the velocity, updated until a relatively unchanging state has been encountered, or until computational limitations are exceeded.

For all iterations, any particle is updated by following two "best" values. The first one is the best solution (fitness) it has achieved so far, referred to as pbest, and the fitness value is also stored. Another "best" value that is tracked by the particle swarm optimizer is the best value obtained so far by any particle in the population, which is called global best (gbest).

In PSO path planning optimization, each particle of a particle swarm represents a single possible path that could be taken by the mobile RFID reader. Hence, the particle swarm attempts to find the optimal path for the mobile RFID reader to move from one node to another. This technique randomly generates a population of particles within the given dimension area. Therefore, the swarm attempts to find the optimal path that will be used for the mobile RFID reader to move to every single node in the given area.

The pseudo code of the PSO algorithm can be summarised as follows:

Step 1 : Initialize parameters $c_{1}, c_{2}$ and $\omega$ for the particles.

Step 2 : Initialize random positions for all dimension each particle and their associated velocities.

Step 3 : Evaluate the fitness function for each particle.

Step 4 : Check criterion termination based on number of iteration.

Step 5 : Update velocities and position based on nearest neighbour constraint.

Step 6 : Update local best (pbest), which compares the current value of fitness function with the previous best value of the particles.

Step 7 : Update global best (gbest), which determines the current global minimum fitness value among the current positions of the particles.

Step 8 : Apply state transition based on nearest neighbour constraint and the process will continue till end condition when maximum number of iterations is reached.

\subsection{Ant Colony Optimization}

The ant colony optimization (ACO) is a meta-heuristic optimization technique for hard combinatorial optimization problems. We examine the ant colony system (ACS) as a representative of the ACO technique. There are three (3) key aspects pertaining to the ACS that should be understood:

a) the state transition rule provides a direct way to balance between exploration of new edges and exploitation of a priori and accumulated knowledge about the problem;

b) the global updating is applied only to edges which belong to the best ant tour; and

c) while ants construct a solution, a local pheromone updating rules applied.

Basically, for the ACS process, $\mathrm{m}$ ants are initially put on $\mathrm{n}$ nodes chosen according to some initialization rule (e.g., randomly). Each ant builds a path by repeatedly applying the state transition rule. During constructing its path, an ant makes some changes to the amount of pheromones on the visited edges by applying the local updating rule. Once all ants have finished their path, the amount of pheromones on edges is modified again by applying the global updating rule. The path with a high amount of pheromones is preferred by the ants, hence, they will choose the short path.

The main goal of the local update is to diversify the search by decreasing the pheromone concentration on the traversed edges. Thus, the ant would choose another route to produce different solutions. This would prevent several ants to produce identical solutions during an iteration. The global pheromone update is applied at the end of each iteration to one ant, which can be either the iteration-best ant or the bestso-far ant. The pheromone updating rules are designed so that they tend to give more pheromone to edges which should be visited by ants. 
The pseudo code of the ACO is as follows:

Step 1 : Initialize parameter $\mathrm{t}, \alpha, \rho, \mathrm{q} 0, \mathrm{nk}$, and calculate $\tau \mathrm{O}$

Step 2 : For every link $(r, s)$, perform the pheromone initialization $\tau(r, s)=\tau_{0}$;

Step 3 : Initialize number of iterations and apply state transition rule based on neighbor constraint

Step 4 : Apply local pheromone update until all ants have built a complete solution

Step 5 : Calculate fitness

Step 6 : Check criterion termination based on number of iteration

Step 7 : Apply global pheromone updating for the best solution produced by ants

Step 8 : Apply state transition based on nearest neighbour constraint and the process will continue till end condition when maximum number of iterations is reached.

\section{SIMULATION SETUP}

Numerous research activities have been proposed in localization and positioning applications of RFID readers and tags [18]. In general, one of the most fundamental issues in wireless sensor networks is reader coverage. Every point of the selected area must be within the sensing range of at least one sensor network. In the literature, solutions to this problem have been proposed in many different ways, e.g., using a mobile reader [7]. The coverage of a wireless sensor network may be approximated by a disk of a prescribed radius (sensing range). Coverage can be computed by taking the union of individual coverage areas of all sensors (readers) in the network. In this experiment, the number of nodes depends on the dimension of the area and interrogation range that we set for the mobile RFID reader. If the dimension of area is large then the number of nodes that a mobile RFID reader traverses is increased. In other words, the interrogation range influences the number of nodes the mobile RFID reader visits.

The size of a hexagon is based the interrogation of a reader. To address this problem, the software simulation that is developed can define the read range of the RFID reader and obstacles inside the area covered, based on user requirement. In this experiment, we start the algorithm with an initialization process to determine the number of readers to be used in a given area, and the system optimizes the number of readers based on the area. Using GA, PSO and ACO techniques, the RFID mobile reader scans every single node inside the area. GA, PSO and ACO attempt to find a path to complete a circle, in which the minimum path (distance) is chosen as the optimal path. We run every algorithm successively for 100 and 500 iterations under the same initialization conditions, then record the minimum and maximum paths for PSO and ACO.

\section{RESULT AND DISCUSSION}

We used the following configuration for the PSO and ACO algorithm. For the PSO algorithm, the number of particle is 10 , the cognition factor $\mathrm{c} 1$ and social factor $\mathrm{c} 2$ are 1.4 , and the inertia weight $\mathrm{w}$ is 0.4 to 0.9 . Whereas for the ACO algorithm, the population (i.e., number of ants) is $10, \rho$ and $\zeta$ are $0.1, \beta$ is 2 and q0 is 0.9. All the algorithms were run 100 and 500 times, as shown in Table 1 and Table 2.

Table 1. The Result of Optimization Path using GA, PSO and ACO for 150 Nodes and 5 Obstacles

\begin{tabular}{ccccccccccccc}
\hline Method & \multicolumn{9}{c}{ GA } & \multicolumn{4}{c}{ PSO } & \multicolumn{4}{c}{ ACO } \\
\hline \# Running & 100 & 500 & 100 & 500 & 100 & 500 & 100 & 500 & 100 & 500 & 100 & 500 \\
\# iteration & 100 & 100 & 500 & 500 & 100 & 100 & 500 & 500 & 100 & 100 & 500 & 500 \\
Max path & 7950.3 & 7966.5 & 7533.1 & 7513.6 & 7938.3 & 7956.4 & 7533.1 & 753.6 & 6999.9 & 6966.9 & 6509.9 & 6314.0 \\
Min Path & 4833.0 & 4791.3 & 4713.2 & 4661.8 & 4721.2 & 4611.8 & 4700.2 & 4661.8 & 4611.8 & 4611.8 & 4661.8 & 4661.8 \\
Avg Path & 5831.6 & 5861.9 & 5524.7 & 5521.4 & 5831.6 & 5861.9 & 5524.7 & 5521.4 & 5613.1 & 5543.4 & 5425.0 & 5221.4 \\
Std Dev & 331.7 & 320.8 & 280.8 & 251.3 & 331.7 & 320.8 & 280.8 & 251.3 & 318.2 & 311.1 & 268.8 & 219.5 \\
\hline
\end{tabular}

Table 2. The Percentage of Optimization Path using GA for 150 Nodes and 5 Obstacles

\begin{tabular}{|c|c|c|c|c|c|c|c|c|}
\hline $\begin{array}{l}\text { \# Running } \\
\text { \# Iteration }\end{array}$ & \multicolumn{2}{|c|}{100} & \multicolumn{2}{|c|}{500} & \multicolumn{2}{|c|}{100} & \multicolumn{2}{|c|}{500} \\
\hline & & $\%$ & & $\%$ & & $\%$ & & $\%$ \\
\hline Uncompleted & 5831 & 58.31 & 28982 & 57.96 & 28493 & 56.99 & 140932 & 56.37 \\
\hline Completed FALSE & 2648 & 26.48 & 12893 & 25.79 & 12093 & 24.19 & 60283 & 24.11 \\
\hline TRUE & 1521 & 8125 & 10298 & 16.25 & 9414 & 18.83 & 48785 & 19.51 \\
\hline Total & 10000 & 100.00 & 50000 & 100 & 50000 & 100 & 250000 & 100 \\
\hline Max Conv at \# & 100 & 100 & $100 \%$ & 0.2 & 489 & 0.98 & 491 & 0.20 \\
\hline Min Conv at \# & 70 & 0.70 & 75 & 0.15 & 150 & 0.30 & 145 & 0.60 \\
\hline Avg Conv & 96.43 & 0.96 & 92.32 & 0.18 & 398.92 & 0.80 & 382.32 & 0.15 \\
\hline Std Dev Conv & 20.91 & & 19.29 & & 290.21 & & 280.25 & \\
\hline
\end{tabular}


The results of the two algorithms show that the ACO algorithm significantly outperformed the PSO algorithm. This demonstrates that the ACO algorithm has faster convergence and adaptability. Simulation results show that for the ACO algorithm, the minimum path (best value) is 4611.82 for 100 runs (and 100 iterations for each run). The completed and true path for ACO is higher than the PSO as shown in Table 3 and Table 4.

Table 3. The Percentage of Optimization Path using PSO for 150 Nodes and 5 Obstacles

\begin{tabular}{ccccccccc}
\hline \# Running & \multicolumn{2}{c}{100} & \multicolumn{2}{c}{500} & \multicolumn{2}{c}{100} & \multicolumn{2}{c}{500} \\
\# Iteration & \multicolumn{2}{c}{100} & $\%$ & \multicolumn{2}{c}{100} & $\%$ & & 500 \\
\hline Uncompleted & 5552 & $55.52 \%$ & 26781 & $53.56 \%$ & 26131 & $52.26 \%$ & 120023 & $48.01 \%$ \\
Completed FALSE & 2401 & $24.01 \%$ & 12921 & $25.84 \%$ & 13393 & $26.79 \%$ & 60283 & $24.11 \%$ \\
TRUE & 2047 & $20.47 \%$ & 10298 & $20.60 \%$ & 10476 & $20.95 \%$ & 69694 & $27.88 \%$ \\
Total & 10000 & $100.00 \%$ & 50000 & $100.00 \%$ & 50000 & $100.00 \%$ & 250000 & $100.00 \%$ \\
Max Conv at \# & 100 & $100 \%$ & 100 & $100 \%$ & 489 & $98 \%$ & 491 & $98 \%$ \\
Min Conv at \# & 60 & $60 \%$ & 60 & $60 \%$ & 125 & $25 \%$ & 147 & $29 \%$ \\
Avg Conv & 90.21 & $90.21 \%$ & 82.32 & $82.32 \%$ & 345.32 & $69.06 \%$ & 320.26 & $64.05 \%$ \\
Std Dev Conv & 15.41 & & 15.34 & & 250.77 & & 250.45 & \\
\hline
\end{tabular}

Table 4. The Percentage of Optimization Path using ACO for 150 Nodes and 5 Obstacles

\begin{tabular}{|c|c|c|c|c|c|c|c|c|c|}
\hline \multirow{2}{*}{\multicolumn{2}{|c|}{$\begin{array}{l}\text { \# Running } \\
\text { \# Iteration }\end{array}$}} & \multicolumn{2}{|c|}{100} & \multicolumn{2}{|c|}{500} & \multicolumn{2}{|c|}{100} & \multicolumn{2}{|c|}{500} \\
\hline & & \multicolumn{2}{|c|}{100} & \multicolumn{2}{|c|}{100} & \multicolumn{2}{|c|}{500} & \multicolumn{2}{|c|}{500} \\
\hline \multirow{2}{*}{\multicolumn{2}{|c|}{ Uncompleted }} & & $\%$ & & $\%$ & & $\%$ & & $\%$ \\
\hline & & 4712 & $47.12 \%$ & 22039 & $44.08 \%$ & 21234 & $42.47 \%$ & 102932 & $41.17 \%$ \\
\hline Completed & TRUE & 2913 & $29.13 \%$ & 14680 & $29.36 \%$ & 15973 & $31.95 \%$ & 83785 & $33.51 \%$ \\
\hline \multicolumn{2}{|c|}{ Total } & 10000 & $100.00 \%$ & 50000 & $100.00 \%$ & 50000 & $100.00 \%$ & 250000 & $100.00 \%$ \\
\hline \multicolumn{2}{|c|}{ Max Conv at \# } & 100 & $100 \%$ & 100 & $100 \%$ & 489 & $98 \%$ & 491 & $98 \%$ \\
\hline \multicolumn{2}{|c|}{ Std Dev Conv } & 15.15 & & 13.44 & & 231.11 & & 213.44 & \\
\hline
\end{tabular}

\section{CONCLUSION}

This paper has presented Solving RFID Mobile Reader Path Problem with Optimization Algorithms. We proposed algorithms for an RFID mobile reader to optimize the path and to cover a given area by putting on the GA, PSO and ACO techniques. These techniques should meet the objective when we set the $n$ to the nearest neighbor in which the mobile reader will not be diverted to a longer path in order to achieve better results. After development of a simulation prototype, and evaluative experimentation of the prototype, the feasibility and effectiveness of the ACO algorithm significantly outperform the GA and PSO algorithm. The results show that the ACO algorithm achieve promising results, and has competitive potential for solving discrete optimization problems like path optimization compared to the PSO algorithm. As recommendation for future work, it would be worthy to further investigate whether the use of both the PSO and ACO algorithms in combination would achieve better accuracy. We plan to further investigate the functionality of this combination approach and present our experimental results in a subsequent paper.

\section{REFERENCES}

[1] P. M. D., "Economic Transformation Programme (ETP) Annual Report," Putrajaya, Jabatan Perdana Menteri, 2012.

[2] Y. B. Gimpilevic and D. A. Savochkin, "Simulation of measuring data obtained from RFID-tags in systems of spatial localization of objects," Springer Journal Computer, 2016.

[3] J. Shen, et al., "A RFID Based Localization Algorithm for Wireless Sensor Networks," Springer Journal Computer, 2016.

[4] A. Ascher, et al., "Localization of UHF RFID Transponders regarding Industry 4.0 Scenarios using Direction of Arrival (DoA) Estimation Techniques," IEEE journal, 2016.

[5] O. Friedewald and M. Lange, "Localization methods by using phase of arrival," IEEE journal, 2016.

[6] F. Seco and A. R. Jim'enez, "RFID-based centralized cooperative localization in indoor environments," IEEE journal, 2016.

[7] Y. Zhao, et al., "VIRE: Active RFID-based localization using virtual reference elimination," Proc. of ICPP, 2007.

[8] X. Huang, et al., "Outdoor localization using active RFID technology," Proc. of BROADNETS, 2006.

[9] S. Chumkamon, et al., Proceedings of ECTI-CON, 2008. A Blind Navigation System Using RFID for Indoor Environments. 
[10] Chou L. D., et al., "Requirement Analysis andImplementation of Palm-based Multimedia Museum Guide Systems," IEEE 18th International, 2004.

[11] F. B. Zahn and C. E. Noon, "Shortest path algorithms: An evaluation using real road networks," Transport. Sci., vol. 32, pp. 65-73, 1998.

[12] G. Desaulniers and F. Soumis, "An efficient algorithm to find a shortest path for a car-like robot," IEEE Trans. Robot Automat., vol/issue: 11(6), pp. 819-828, 1995.

[13] J. Moy, "Open Shortest Path First Version 2. RFQ 1583," Internet Engineering Task Force, 1994.

[14] S. Anusha and S. Iyer, "A Coverage Planning Tool for RFID Networks With Mobile Readers: EUC workshops," LNCS 3823, 2005.

[15] A. W. Mohemmed, et al., "Solving shortest path problem using particle swarm optimization," Applied Soft Computing, vol. 8, pp. 1643-1653, 2008.

[16] J. B. Orlin, et al., "A faster algorithm for the single source shortest path problem with few distinct positive lengths," Journal of Discrete Algorithms, vol/issue: 8(2), pp. 189-198, 2010.

[17] J. Kennedy and R. C. Eberhart, "Particle swarm optimization," Proceedings of the IEEE International Conference on Neural Networks, pp. 1942-1948, 1995.

[18] D. E. Goldberg, "Genetic Algorithms in Search, Optimization and Machine Learning," Addison-Wesley, Reading, MA, 1989. 